\title{
INFLUENCE OF TIME DELAYS ON THE HAHNFELDT ET AL. ANGIOGENESIS MODEL DYNAMICS
}

Abstract. We study the influence of time delays on the dynamics of the general Hahnfeldt et al. model of an angiogenesis process. We analyse the dynamics of the system for different values of the parameter $\alpha$ which reflects the strength of stimulation of the vessel formation process. Time delays are introduced in three subprocesses: tumour growth, stimulation and inhibition of vessel formation (represented by endothelial cell dynamics). We focus on possible destabilisation of the positive steady state due to the delay. Results are illustrated by numerical simulations performed for parameter values estimated by Hahnfeldt et al. for tumour volume data of Lewis lung carcinoma implanted in mice.

1. Introduction. Tumour angiogenesis is one of the most important processes in tumour dynamics. It allows solid tumours to grow to sizes exceeding 2-3 mm, which is a typical maximal diameter for avascular tumours. Furthermore, it is necessary for tumour metastasis. From the medical treatment point of view, it is considered as a target for chemotherapy, which was discovered by Folkman in 1972 ([11]). Today, we are able to understand and describe this process much better (see e.g. [3]). In the absence of nutrients, tumour cell secrete growth factors, which can stimulate endothelial cells to proliferate, migrate and form new blood vessels. However, in [3] it is claimed that the newly formed vessels have highly unstable structure and its stabilisation crucially depends on maturation. Therefore, the effective vessel density EVD (that is, the total perfused vasculature) can exhibit an oscillatory behaviour and it can be connected with the delays present in the subprocesses of the angiogenesis process considered. Thus, the influence of time delays on the angiogenesis dynamics can be an interesting research topic.

2000 Mathematics Subject Classification: 92C60, 37G15, 34K20, 34K60.

Key words and phrases: HIV-1 infection, cancer, steady state, stability, Hopf bifurcation, time delay. 
The process of angiogenesis is complicated, and mathematical modelling can bring a better understanding and lead to better treatment protocols (see [3]). The basic mathematical model of this process was proposed by Hahnfeldt et al. [19]. It is described by a system of two ODEs with the Gompertzian type tumour growth and with carrying capacity equal to endothelial cell volume. This model was studied in [24]. On the basis of the Hahnfeldt et al. model some treatment protocols have been proposed (see e.g. $[10,23,26])$. Another, much more complicated numerical model was proposed in [3] and compared with experimental data in [2]. Due to the complexity of that model, in [1] a simple model (more precisely, a family of models) was proposed. This model was analysed in [1] and [14]. Simplification of this model and some basic comparison with the Hahnfeldt et al. model was presented in [13]. A model combining the ideas of carrying capacity depending on vessel density with [1] was proposed in [7]. On the other hand, in [5] some preliminary analysis of the influence of time delays on the Hahnfeldt et al. model dynamics was performed.

In this paper we follow the ideas of [5]. We analyse the dynamics of the system for different values of the parameter $\alpha$ which reflects the strength of stimulation of the vessel formation process. In [19] this parameter was chosen as $\alpha=1$, while in [10], $\alpha=0$. We study the dynamics of the general Hahnfeldt et al. model (that is, for any $\alpha \in[0,1]$ ) with respect to the magnitude of one of the delays introduced. The results are illustrated by numerical simulations for the model parameters estimated in [19] for tumour volume data of Lewis lung carcinoma implanted in mice, while in [5] simulations were performed for arbitrary chosen parameters.

2. Presentation of the model. The Hahnfeldt et al. model [19] was built on the basis of Gompertzian growth of tumour cells, that is,

$$
\dot{p}=-r p \ln \left(\frac{p}{K}\right),
$$

where $p$ denotes the tumour cell density and $K$ is the maximal tumour size (carrying capacity in terms of population dynamics). The Gompertz model ([18]) was widely used in tumour modelling (see also [4, 6, 15, 21, 22, 25, 27] and references therein).

In the Hahnfeldt et al. model it is assumed that $K$ is non-constant and depends on the vessel volume which is represented by the endothelial cell density $q$. The endothelial cell dynamics depends on their production stimulated by tumour cells, inhibition due to the presence of mature vessels, and the natural death process. In [19] it is assumed that the stimulation function has the form $p^{\alpha} q^{\beta}$ with $\alpha+\beta=1$. Hence, in the general case the stimulation term takes the form $p^{\alpha} q^{1-\alpha}$ with $\alpha \in[0,1]$. Finally, in [19] it is assumed 
that $\alpha=1$, while in [10], $\alpha=0$ was chosen. In this paper we would like to compare the behaviour of the system for different values of $\alpha$.

The inhibition process is described by the term $p^{2 / 3} q$, where the exponent $2 / 3$ reflects the ratio of the tumour surface to its volume.

Hence, in the general case the system of equations describing the Hahnfeldt et al. model has the following form:

$$
\begin{aligned}
& \dot{p}=-r p \ln \left(\frac{p}{q}\right), \\
& \dot{q}=b p^{\alpha} q^{1-\alpha}-\left(a p^{2 / 3}+\mu\right) q,
\end{aligned}
$$

with positive coefficients. However, $\mu$ is very small compared to other parameters (see e.g. [23]), so that $\mu \approx 0$. Therefore, in the following we assume that $\mu=0$ for simplicity.

Studying the behaviour of the system (1) we follow the ideas presented in [1]. We change variables so that $u=p / q$ and introduce time delays to the processes described in the model. The first delay $\tau_{1}$ reflects the delay of tumour cell production with respect to stimulus. The same delay appears in the first term of the second equation, which is connected with the change of variables (in the original system the logarithmic term is absent in the second equation of (1)). The second delay $\tau_{2}$ reflects the delay of tumour vessel production with respect to stimulus. In [1] this delay appears in the second term of the second equation. However, in our case this term describes two different processes, namely stimulation and inhibition. Therefore, we can consider two delays instead of one. In the new variables the system with time delays reads

$$
\begin{aligned}
\dot{p}(t) & =-r p(t) \ln u\left(t-\tau_{1}\right), \\
\dot{u}(t) & =-u(t)\left(r \ln u\left(t-\tau_{1}\right)+b\left(u\left(t-\tau_{2}\right)\right)^{\alpha}-a\left(p\left(t-\tau_{3}\right)\right)^{2 / 3}\right),
\end{aligned}
$$

for $u, p>0$, with $\tau_{i} \geq 0, i=1,2,3$.

We focus on the behaviour of the solutions to (2) for different $\alpha \in[0,1]$ and other parameters estimated in [19]. After rescaling the variable $p$ by $10^{-4}$ these parameters have the following values:

$$
r=0.192, \quad a=4.052, \quad b=5.85 .
$$

In [19] the value $\alpha=1$ was chosen, and therefore in the following we refer to (2) with $\alpha=1$ as the Hahnfeldt et al. model, while for $\alpha=0$ we talk of the Ergun et al. model due to the value $\alpha=0$ chosen in [10]. For comparison we use also the medium value $\alpha=1 / 2$.

3. Asymptotic stability analysis. In this section we study the behaviour of the system (2) for different $\alpha \in[0,1]$. We start from the case without delay, that is, $\tau_{i}=0$ for $i=1,2,3$. We analyse (2) in the extended 
phase space $\{(p, u): u>0, p \geq 0\}$. It is easy to see that this system has one positive steady state $\left(p^{*}, u^{*}\right)$ with $u^{*}=1$ and $p^{*}=(b / a)^{3 / 2}$, and in the extended phase space there exists a semi-trivial steady state with $\bar{p}=0$ and $\bar{u}$ defined implicitly by $r \ln \bar{u}+b \bar{u}^{\alpha}=0$. Uniqueness of the semi-trivial steady state follows from monotonicity of both terms in this formula.

Theorem 1. For every $\alpha \in[0,1]$ the positive steady state $\left(p^{*}, u^{*}\right)$ of $(2)$ is globally stable in $\left(\mathbb{R}^{+}\right)^{2}$. For $\alpha \geq 2 / 3$ this state is a stable node, while for $\alpha<2 / 3$ it is a node or focus depending on the value of $r$ : for $r \in\left(\frac{(4-3 \alpha) b}{3}-\frac{\sqrt{\Delta}}{2}, \frac{(4-3 \alpha) b}{3}+\frac{\sqrt{\Delta}}{2}\right)$, where $\Delta=\frac{4}{9} b^{2}(3 \alpha-4)^{2}-4 \alpha^{2} b^{3}$, it is a stable focus, otherwise it is a stable node.

Proof. It is easy to see that the characteristic polynomial for (2) is

$$
W(\lambda)=\lambda^{2}+(r+\alpha b) \lambda+\frac{2}{3} r b
$$

for every $\alpha \in[0,1]$. Its discriminant $\Delta_{W}=r^{2}+\frac{2}{3} r b(3 \alpha-4)+\alpha^{2} b^{2}$ is always non-negative for $\alpha \geq 2 / 3$, which means that $W(\lambda)$ has only real roots and they are negative. Hence, the positive steady state is a stable node in this case. For $\alpha<2 / 3$ the sign of this discriminant depends on the roots of $\Delta_{W}$ as a function of $r$, that is, $r_{1,2}=\left(\frac{2}{3}(4-3 \alpha) b \pm \sqrt{\Delta}\right) / 2$. For $r \in\left(r_{1}, r_{2}\right)$ we have $\Delta_{W}<0$, that is, the positive steady state is a stable focus. Otherwise it is a stable node.

Studying the phase portrait (see Fig. 1), we see that all orbits are bounded independently of $\alpha \in[0,1]$. Therefore, using the Poincaré-Bendixson theorem we conclude that each orbit either tends to the positive steady state or to the closed orbit surrounding it. However, the Dulac-Bendixson criterion for (2) with the standard function for Lotka-Volterra systems (see e.g. [17] where it was used for the system with switching functions) $B(p, u)=1 / p u$ implies that there are no closed orbits in $\left(\mathbb{R}^{+}\right)^{2}$. Hence, all solutions to $(2)$ with positive initial data tend to $\left(p^{*}, u^{*}\right)$ as $t \rightarrow \infty$.
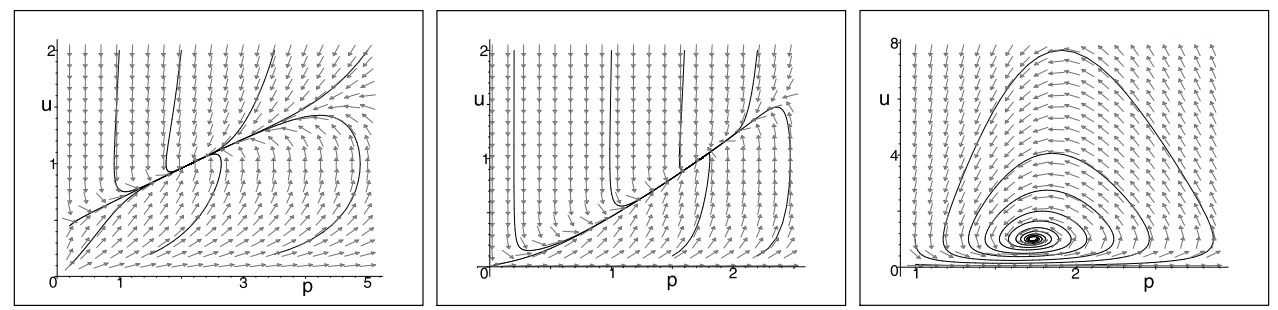

Fig. 1. Phase space portraits for (2) with parameter values from [19] and different $\alpha$ : $\alpha=1$ (left), $\alpha=1 / 2$ (middle) and $\alpha=0$ (right) 
Studying the character of the semi-trivial steady state we see that for $u \rightarrow 0$ and every $p>0$ we have $\dot{p} \rightarrow+\infty$. Thus the orbits are parallel to the horizontal axis at the points $(p, 0)$, and $\dot{p}>0$ for small positive $u$. For $p=0$ we have $u \rightarrow \bar{u}$. This shows that the semi-trivial steady state is a saddle point.

3.1. Influence of time delays. In this subsection we study the influence of delays on the dynamics of system (2). We focus on the simplest cases when only one of the delays is non-zero or some two delays are equal positive. It is known (see e.g. [12]) that if the steady state is a saddle for $\tau=0$, then it cannot gain stability for positive delays. Therefore, we only study the positive steady state $\left(p^{*}, u^{*}\right)$. We calculate the characteristic quasi-polynomial. In the general case it has the form

$$
W_{\tau}(\lambda)=\lambda^{2}+\lambda\left(r e^{-\lambda \tau_{1}}+\alpha b e^{-\lambda \tau_{2}}\right)+\frac{2}{3} r b e^{-\lambda \tau_{1}} e^{-\lambda \tau_{3}} .
$$

We know that stability depends continuously on the magnitude of the delay. Therefore, to obtain destabilisation, the existence of purely imaginary characteristic values is necessary. Hence, in the following we analyse $W_{\tau}(\lambda)$ for $\lambda=i \omega$ looking for its roots and the global change of the argument $\Delta \arg _{\omega \in[0, \infty)} W_{\tau}(i \omega)$ which determines stability due to the Mikhailov or Nyquist criterion (see e.g. $[12,20]$ ).

We illustrate the results of analytical investigations with numerically computed solutions for the parameter values from [19].

Because we study (2) with only one positive delay, the characteristic quasi-polynomial (3) for $\lambda=i \omega$ has a general form

$$
W_{\tau}(i \omega)=-\omega^{2}+c_{1} i \omega+c_{2}+\left(d_{1} i \omega+d_{2}\right) \exp (-i \omega \tau),
$$

where $\tau=\tau_{1}, \tau_{2}, \tau_{3}$. Purely imaginary roots of $W_{\tau}$ exist iff $\|-\omega^{2}+c_{1} i \omega$ $+c_{2}\left\|^{2}=\right\| d_{1} i \omega+d_{2} \|^{2}$. Following the ideas from $[9,8]$ and defining the auxiliary function

$$
F(x)=x^{2}+\left(c_{1}^{2}-2 c_{2}-d_{1}^{2}\right) x+c_{2}^{2}-d_{2}^{2}, \quad x=\omega^{2},
$$

we see that the existence of purely imaginary eigenvalues is equivalent to the existence of positive roots of $F$. If $F$ has a positive root $x_{0}$, then there exists a pair $\pm i \omega_{0}$ of purely imaginary eigenvalues with $\omega_{0}=\sqrt{x_{0}}$. For this $\omega_{0}$ we find a sequence $\left\{\tau^{n}\right\}$ of delays for which the switch of stability can occur. This sequence is defined implicitly by its sine and cosine:

$$
\sin \left(\tau^{n} \omega_{0}\right)=\omega_{0} \frac{c_{1} d_{2}-c_{2} d_{1}+d_{1} \omega_{0}^{2}}{d_{2}^{2}+d_{1}^{2} \omega_{0}^{2}}, \quad \cos \left(\tau^{n} \omega_{0}\right)=\frac{\left(d_{2}-c_{1} d_{1}\right) \omega_{0}^{2}-c_{2} d_{2}}{d_{2}^{2}+d_{1}^{2} \omega_{0}^{2}}
$$

Typically, the system looses stability for the smallest $\tau^{n}$, that is, $\tau^{0} \in(0,2 \pi)$, satisfying the relations above. 
Case $\tau_{1}>0$ and $\tau_{2}=\tau_{3}=0$. In this case the coefficients of the characteristic quasi-polynomial (4) are equal to

$$
c_{1}=\alpha b, \quad c_{2}=0, \quad d_{1}=r, \quad d_{2}=\frac{2}{3} b r,
$$

and the free term of the auxiliary function $F$ is $c_{2}^{2}-d_{2}^{2}=-\frac{4}{9} b^{2} r^{2}<0$. This implies that $F$ has a unique positive root, and therefore $W_{\tau}$ has exactly one pair of purely imaginary roots. Hence, there exists a unique pair of purely imaginary eigenvalues $\pm i \omega_{0}$, and a sequence $\left\{\tau_{1}^{n}\right\}$ of critical values of the delay $\tau_{1}$ for which $\left(p^{*}, u^{*}\right)$ can loose stability. In Figs. 2 and 3 we
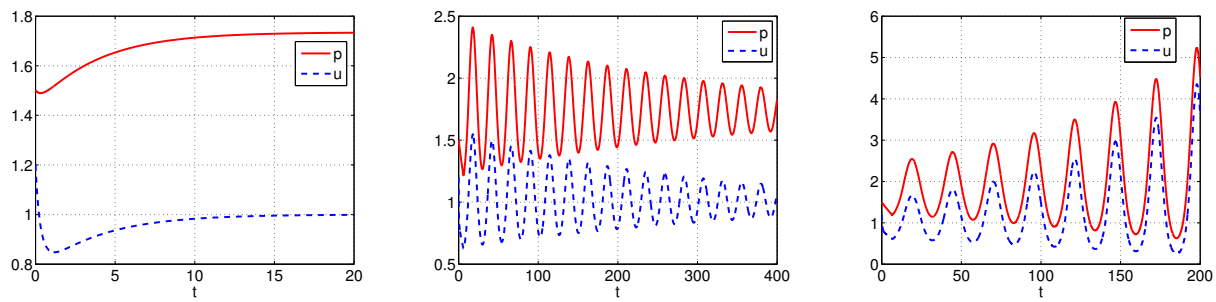

Fig. 2. Solutions to (2) for $\alpha=1 / 2$ and for $\tau_{1}=0.1$ (left), $\tau_{1}=5.9$ (middle) and $\tau_{1}=6.4$ (right)
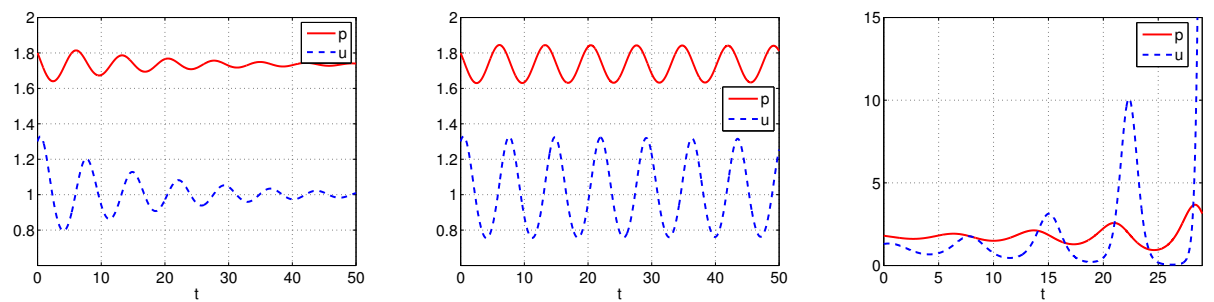

Fig. 3. Solutions to (2) for $\alpha=0$ and for $\tau_{1}=0.1$ (left), $\tau_{1}=0.25$ (middle) and $\tau_{1}=0.5$ (right)

present examples of the solutions to (2) in this case for $\alpha=1 / 2$ and $\alpha=0$, respectively. It can be observed that destabilisation of the positive steady state occurs. The Mikhailov or Nyquist criterion used for this data shows that destabilisation takes place for $\tau_{1} \approx 0.2522$ for $\alpha=0$ (the Ergun et al. model), $\tau_{1} \approx 6.06$ for $\alpha=1 / 2$. The left-hand side graph in Fig. 8 shows the dependence of the critical value $\tau_{1 \text {,crit }}$ on the parameter $\alpha$. The results of simulations suggest that system (2) exhibits Hopf bifurcation for every $\alpha \in[0,1]$. Exactly the same qualitative behaviour can be observed for the Hahnfeldt et al. model. The difference is only quantitative - the threshold value of delay changes. In this case the destabilisation takes place at $\tau_{1} \approx$ 12.36. It should be marked that the threshold value of delay for the Hahnfeldt et al. model is large and can be irrelevant from the biological point of view. 
Case $\tau_{2}>0$ and $\tau_{1}=\tau_{3}=0$. Now,

$$
c_{1}=r, \quad c_{2}=\frac{2}{3} b r, \quad d_{1}=\alpha b, \quad d_{2}=0 .
$$

In this case the free term of $F$ is positive $\left(c_{2}^{2}-d_{2}^{2}=4 / 3 r^{2} b^{2}>0\right)$, and therefore the existence of positive roots of $F$ depends on the signs of the coefficient $c_{1}^{2}-2 c_{2}-d_{1}^{2}$ of the linear term and the discriminant of $F$. It should be noticed that in [5] there is a mistake because it is assumed that this coefficient is always positive.

TheOREM 2. If $\tau_{2}>0$ while $\tau_{1}=\tau_{3}=0$, then the positive steady state $\left(p^{*}, u^{*}\right)$ is locally asymptotically stable independently of the magnitude of $\tau_{2}$ for $r>\alpha b$. If $r \leq \alpha b$, then there exists a critical value of the delay $\tau_{2}$ for which this state can loose stability.

Proof. Due to the form of $F$, if $c_{1}^{2}-2 c_{2}-d_{1}^{2}=r^{2}-\frac{4}{3} b r-\alpha^{2} b^{2} \geq 0$, then $F$ can have either real negative roots or complex roots with negative real part. This implies that there are no purely imaginary eigenvalues of (2) for $r \geq\left(2+\sqrt{9 \alpha^{2}+4}\right) b / 3$. On the other hand, if $r<\left(2+\sqrt{9 \alpha^{2}+4}\right) b / 3$, then $\Delta_{F}=\left(r^{2}-\frac{4}{3} b r-\alpha^{2} b^{2}\right)^{2}-\frac{16}{9} b^{2} r^{2}$ and $\Delta_{F}<0$ for $r>\alpha b$, while $\Delta_{F} \geq 0$ for $r \leq \alpha b$. Hence, for $r \leq \alpha b$ the auxiliary function $F$ has positive roots, while for $r>\alpha b$ positive roots do not exist.

REMARK 1. For the Hahnfeldt et al. model and parameter values estimated in [19] we have $\alpha=1$ and $r<b$, which implies that $\left(p^{*}, u^{*}\right)$ can loose stability, while for the Ergun et al. model, always $r>\alpha b=0$, implying that the positive steady state is locally asymptotically stable independently of the magnitude of $\tau_{2}$. It should also be noticed that for the parameter $\alpha=1 / 2$ chosen for comparison in this paper and other parameters from [19] we have $r<\alpha b=b / 2$.
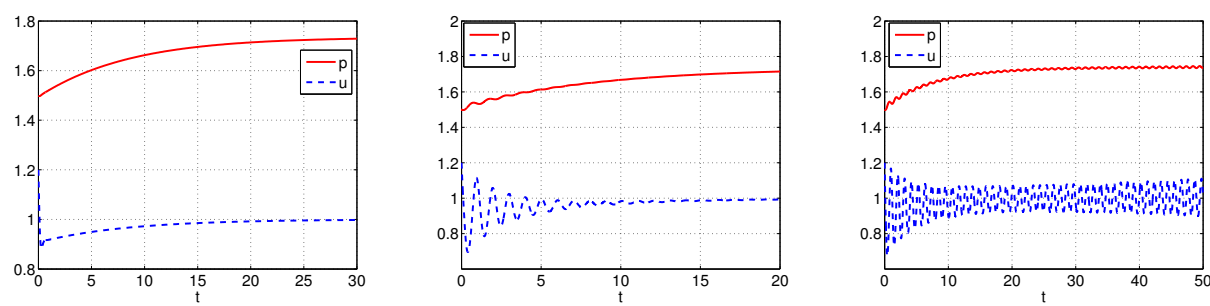

Fig. 4. Solutions to (2) for $\alpha=1$ and for $\tau_{2}=0.1$ (left), $\tau_{2}=0.25$ (middle) and $\tau_{2}=0.27$ (right)

Examples of the behaviour of solutions to the Hahnfeldt et al. model in this case are presented in Fig. 4. With an increasing delay, solutions exhibit an oscillatory behaviour. Bifurcation occurs at $\tau_{2} \approx 0.265$. As in the previous case, this dynamics suggests Hopf bifurcation. The behaviour of the solutions 
to (2) for $\alpha=1 / 2$ is similar - there is only quantitative difference in the threshold value of the delay. Hopf bifurcation occurs at $\tau_{2} \approx 0.518$. In the middle graph of Fig. 8 the dependence of the bifurcation threshold on $\alpha$ is presented.

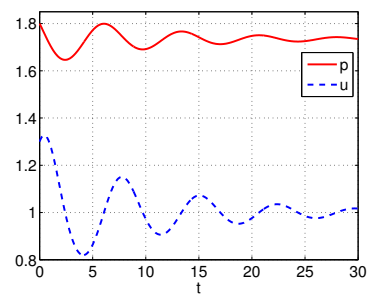

Fig. 5. Solution to (2) for $\alpha=0$

REMARK 2. In this case the dynamics of the Ergun et al. model does not involve time delay. Therefore, for all $\tau_{2}>0$ solutions have the same dynamics. In Fig. 5 a sample solution is presented.

Case $\tau_{3}>0$ and $\tau_{1}=\tau_{2}=0$. In this case,

$$
c_{1}=r+\alpha b, \quad c_{2}=0, \quad d_{1}=0, \quad d_{2}=\frac{2}{3} b r
$$

and the free term of $F$ is equal to $c_{2}^{2}-d_{2}^{2}=-\frac{4}{9} b^{2} r^{2}<0$. As before, the positive steady state can loose stability. In Fig. 6 examples of the behaviour of
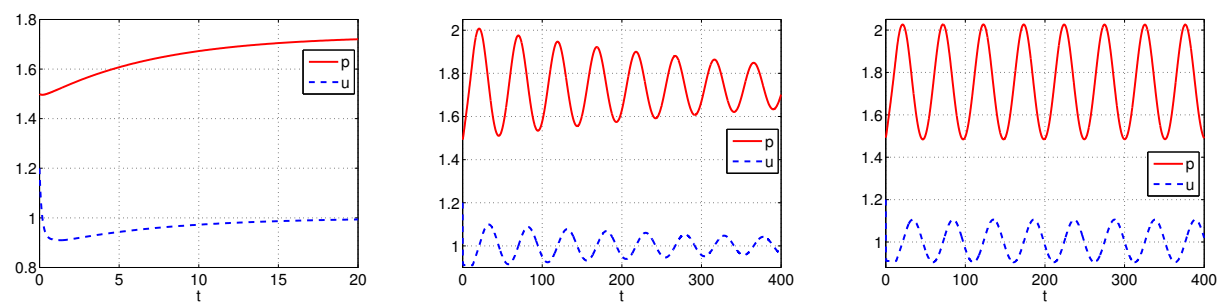

Fig. 6. Solutions to (2) for $\alpha=1$ and for $\tau_{3}=1.0$ (left), $\tau_{3}=12$ (middle) and $\tau_{3}=12.51$ (right)

solutions to the Hahnfeldt et al. model are presented. Again, computer simulations show that for some values of parameters, non-dumping oscillations arise. This suggests a loss of stability of the steady state and occurrence of Hopf bifurcation. The Nyquist criterion used for this data shows that destabilisation takes place for $\tau_{3} \approx 12.51$. For the case $\alpha=1 / 2$ and for the Ergun et al. model, similar qualitative behaviour is observed. However, the bifurcation thresholds differ and have the following values: $\tau_{3} \approx 6.237$ for $\alpha=1 / 2$ and $\tau_{3} \approx 0.2585$ for $\alpha=0$. Similarly to the first case with $\tau_{1}>0$ and 
$\tau_{2}=\tau_{3}=0$, we see that the threshold value of the delay for the Hahnfeldt et al. model is large.

Case $\tau_{2}=\tau_{3}>0$ and $\tau_{1}=0$. Following the ideas presented in [1] we can consider the additional case when $\tau_{2}=\tau_{3}>0$. In this case

$$
c_{1}=r, \quad c_{2}=0, \quad d_{1}=\alpha b, \quad d_{2}=\frac{2}{3} b r
$$

and the free term is the same as in the previous case, which obviously implies that a change of stability is possible. The qualitative behaviour of solutions is very similar to the case with $\tau_{1}=\tau_{2}=0$ and $\tau_{3}>0$. Numerical simulations show a change of stability and suggest that Hopf bifurcation occurs. The Nyquist criterion used for this data shows that destabilisation takes place for $\tau_{2}=\tau_{3} \approx 0.27$ for the Hahnfeldt et al. model, at $\tau_{2}=\tau_{3} \approx 0.528$ for the case $\alpha=1 / 2$, and at $\tau_{2}=\tau_{3} \approx 0.258$ for the Ergun et al. model.

General case. As is easily seen from the previous subsections, in the general case the positive steady state can be destabilised by delays, independently of other model parameters. Moreover, in that case, stability switches can occur (see e.g. [16]). In Fig. 7 we may observe that increasing delay
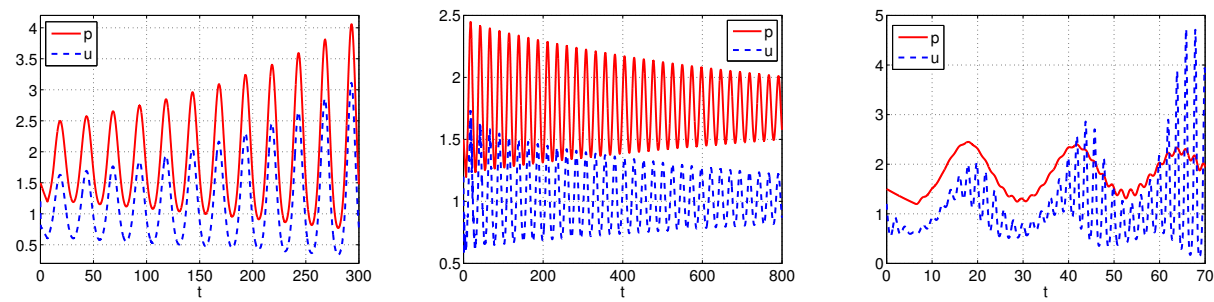

Fig. 7. Solutions to (2) for $\alpha=1 / 2$ and $\tau_{1}=6.4, \tau_{3}=0$ and $\tau_{2}=0.2$ (left), $\tau_{2}=0.46$ (middle) and $\tau_{2}=0.49$ (right)

leads to stabilisation of the steady state. For fixed $\tau_{1}=6.4$ and $\tau_{3}=0$, and small $\tau_{2}$, solutions to (2) exhibit oscillations whose amplitude increases (Fig. 7, left). After increasing the delay, the amplitude is decreasing in time (Fig. 7, middle). After further increasing the delay, oscillations arise again (Fig. 7, right). Using the Nyquist criterion we may approximate points at which stability switches occur. For the data used in simulations it turns out that for small $\tau_{2}<0.29$ the steady state is unstable, for $0.29<\tau_{2}<0.47$ it is stable, and for $\tau_{2}>0.47$ it is unstable. We deduce that stability switches occur around $\tau_{2}=0.29$ and $\tau_{2}=0.47$.

4. Conclusions. We have presented a modified Hahnfeldt et al. model. We consider the case of $\alpha \in[0,1]$, while in [19] this parameter was chosen to be $\alpha=1$. On the other hand, in [10] the authors used $\alpha=0$. We rewrite the original model in such a way that system (2) is similar to systems presented 

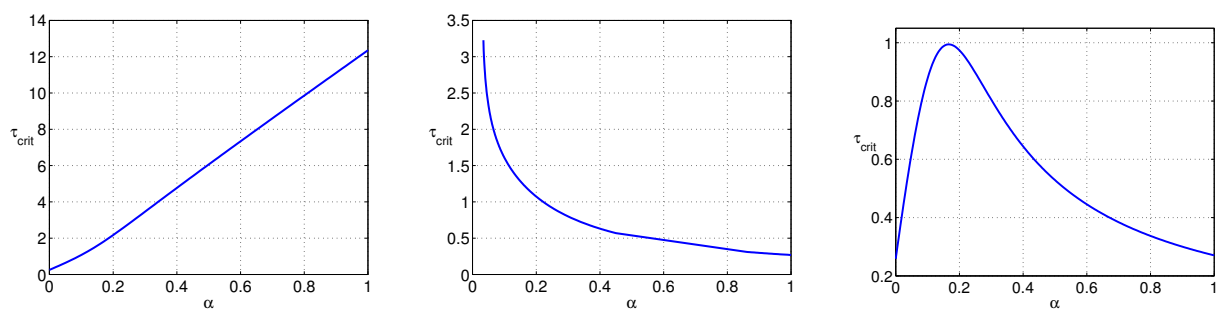

Fig. 8. Dependence of the critical value $\tau_{\text {crit }}$ where destabilisation occurs on the parameter $\alpha$ in different cases. Left: $\tau_{1}>0, \tau_{2}=\tau_{3}=0$; middle: $\tau_{1}=0, \tau_{2}>0$ and $\tau_{3}=0$; right: $\tau_{1}=0, \tau_{2}=\tau_{3}>0$

in $[3,1,2]$ and analysed in $[14,13]$. However, there is a difference between our model and those from $[3,1,2,14,13]$. We use the new variable $u=p / q$, while the models proposed in [1] are formulated in terms of EVD which is equal to $q / p=1 / u$. We have decided to use $u$ instead of EVD because this allows us to consider the extended phase space with $p=0$.

We have introduced time delays in three terms and carried out a simple stability analysis. We have shown that a time delay in the stimulus term (i.e. $\left.\tau_{2}\right)$ for some values of the model parameters cannot lead to destabilisation (as in the Ergun et al. model), while delays in other terms may lead to destabilisation. Biologically, this suggests that the stimulation process is very strong itself, so that the delay introduced into it does not significantly influence the system dynamics. Numerical simulations show that a loss of stability really occurs (see Figs. 2-4, 6, 7). The simulations suggest that system (2) is more sensitive to the delay $\tau_{2}$ of the vessel production than to the others. Destabilisation occurs for smaller delays (see Figs. 4, 7 for $\tau_{2}>0$ and Figs. 2, 3, 6 for $\tau_{2}=0$ ). Stability switches are also possible. However, the computed threshold values of delays (namely $\tau_{1}$ when $\tau_{2}=\tau_{3}=0$ and $\tau_{3}$ when $\tau_{1}=\tau_{2}=0$ ) suggest that all delays should be taken into account, especially in the case of the Hahnfeldt et al. model, where these values seem to be biologically irrelevant compared to the case $\tau_{1}=\tau_{3}=0$.

Acknowledgements. The paper was supported by Polish Ministry of Science, grant No. 1P03A 02830.

\section{References}

[1] Z. Agur, L. Arakelyan, P. Daugulis and Y. Ginosar, Hopf point analysis for angiogenesis models, Disrete Contin. Dynam. Systems B 4 (2004), 29-38.

[2] L. Arakelyan, Y. Merbl and Z. Agur, Vessel maturation effects on tumour growth: validation of a computer model in implanted human ovarian carcinoma spheroids, Eur. J. Cancer 41 (2005), 159-167. 
[3] L. Arakelyan, B. Vainstein and Z. Agur, A computer algorithm describing the process of vessel formation and maturation, and its use for predicting the effects of antiangiogenic and anti-maturation therapy on vascular tumor growth, Angiogenesis 5 (2002), 203-214.

[4] I. D. Bassukas, Comparative Gompertzian analysis of alterations of tumor growth patterns, Cancer Res. 54 (1994), 4385-4392.

[5] M. Bodnar and U. Foryś, Hahnfeldt angiogenesis model with time delays, in: Proc. 13th National Conference on Applications of Mathematics in Biology and Medicine, Serpelice, 2007, 18-23.

[6] —, - Three types of simple DDEs describing tumour growth, J. Biol. Systems 14 (2007), 453-471.

[7] - - - Angiogenesis model with carrying capacity depending on vessel density, ibid. 17 (2009), 1-25.

[8] K. L. Cooke and P. van den Driessche, On zeroes of some transcendental equations, Funkcial. Ekvac. 29 (1986), 77-90.

[9] K. L. Cooke and Z. Grossman, Discrete delay, distributed delay and stability switches, J. Math. Anal. Appl. 86 (1982), 592-627.

[10] A. Ergun, K. Camphausen and L. M. Wein, Optimal scheduling of radiotherapy and angiogenic inhibitors, Bull. Math. Biol. 65 (2003), 407-424.

[11] J. Folkman, Tumor angiogenesis: Therapeutic implications, N. English J. Med. 285 (1971), 1182-1186.

[12] U. Foryś, Biological delay systems and the Mikhailov criterion of stability, J. Biol. Systems 12 (2004), 1-16.

[13] - Two dimensional cancer angiogenesis model, in: Proc. 12th National Conference on Applications of Mathematics in Biology and Medicine, Koninki, 2006, 53-58.

[14] U. Foryś, Y. Kheifetz and Y. Kogan, Critical-point analysis for three-variable cancer angiogenesis models, Math. Biosci. Engrg. 2 (2005), 511-525.

[15] U. Foryś and A. Marciniak-Czochra, Logistic equation in tumour growth modelling, Int. J. Appl. Math. Comput. Sci. 13 (2003), 317-325.

[16] U. Foryś and M. J. Piotrowska, Time delays in solid avascular tumour, in: Proc. 10th National Conference on Applications of Mathematics in Biology and Medicine, Święty Krzyż, 2004, 43-48.

[17] U. Foryś, J. Waniewski and P. Zhivkov, Anti-tumor immunity and tumor antiimmunity in a mathematical model of tumor immunotherapy, J. Biol. Systems 14 (2006), 13-30.

[18] B. Gompertz, On the nature of the function expressive of the law of human mortality, and on a new mode of determining the value of life contingencies, Philos. Trans. Roy. Soc. 115 (1825), 513-585.

[19] P. Hahnfeldt, D. Panigrahy, J. Folkman and L. Hlatky, Tumor development under angiogenic signaling: a dynamical theory of tumor growth, treatment response, and postvascular dormancy, Cancer Res. 59 (1999), 4770-4775.

[20] Y. Kuang, Delay Differential Equations with Application in Population Dynamics, Academic Press, Boston, 1993.

[21] A. K. Laird, Dynamics of tumour growth, British J. Cancer 18 (1964), 490-502.

[22] - Dynamics of tumour growth: comparison of growth rates and extrapolation of growth curve to one cell, ibid. 19 (1965), 278-291.

[23] U. Ledzewicz and H. Schättler, Anti-angiogenic therapy in cancer treatment as an optimal control problem, SIAM J. Control Optim. 46 (2007), 1053-1079.

[24] A. d'Onofrio and A. Gandolfi, Tumour eradication by antiangiogenic therapy: analysis and extensions of the model by Hahnfeldt et al. (1999), Math. Biosci. 191 (2004), 159-184. 
[25] J. A. O'Donoghue, The response of tumours with Gompertzian growth characteristics to fractionated radiotherapy, Int. J. Radiat. Biol. 72 (1997), 325-339.

[26] A. Świerniak, A. Gala, A. Gandolfi and A. d'Onofrio, Optimization of anti-angiogenic therapy as optimal control problem, in: Proc. IASTED Biomechanics 2006, Actapress, 2006.

[27] T. E. Wheldon, Mathematical Models in Cancer Research, Hilger, Boston, 1988.

Institute of Applied Mathematics \& Mechanics

Faculty of Mathematics, Informatics \& Mechanics

University of Warsaw

Banacha 2

02-097 Warszawa, Poland

E-mail: mbodnar@mimuw.edu.pl urszula@mimuw.edu.pl

Received on 3.6.2008;

revised version on 11.9.2008 